\title{
GLOBAL ANALYSIS OF PIECEWISE LINEAR SYSTEMS USING IMPACT MAPS AND QUADRATIC SURFACE LYAPUNOV FUNCTIONS
}

\author{
Jorge M. Gonçalves* , Alexandre Megretski ${ }^{\dagger}$, Munther A. Dahleh ${ }^{\dagger}$ \\ ${ }^{*}$ California Institute of Technology \\ e-mail: jmg@cds.cal tech.edu \\ http: / / www.cds.caltech.edu/ jmg/ \\ ${ }^{\dagger}$ Massachusetts Institute of Technology \\ e-mail: ameg@mit.edu, dahleh@mit.edu
}

Keywords: hybrid systems, stability analysis, robustness analysis, performance analysis, switching surfaces.

\begin{abstract}
In this paper we develop an entirely new constructive global analysis methodology for a class of hybrid systems known as Piecewise Linear Systems (PLS). This methodology consists in inferring global properties of PLS solely by studying their behavior at switching surfaces associated with PLS. The main idea is to analyze impact maps, i.e., maps from one switching surface to the next switching surface, by constructing quadratic Lyapunov functions on switching surfaces. We found that an impact map induced by an LTI flow between two switching surfaces can be represented as a linear transformation analytically parameterized by a scalar function of the state. This representation of impact maps allows the search for quadratic surface Lyapunov functions to be done by simply solving a set of LMIs. Global asymptotic stability, robustness, and performance of limit cycles and equilibrium points of PLS can this way be efficiently checked. These new results were successfully applied to certain classes of PLS. Although this analysis methodology yields only sufficient criteria of stability, it has shown to be very successful in globally analyzing a large number of examples with a locally stable limit cycle or equilibrium point. In fact, it is still an open problem whether there exists an example with a globally stable limit cycle or equilibrium point that cannot be successfully analyzed with this new methodology. Examples analyzed include systems of relative degree larger than one and of high dimension, for which no other analysis methodology could be applied.
\end{abstract}

\section{Introduction}

In this work we are interested in a class of nonlinear systems known as piecewise linear systems (PLS). PLS are characterized by a finite number of linear dynamical models together with a set of rules for switching among these models. Therefore, this model description causes a partitioning of the state space into cells. These cells have distinctive properties in that the dynamics within each cell are described by linear dynamic equations. The boundaries of each cell are in effect switches between different linear systems. Those switches arise from the breakpoints in the piecewise linear functions of the model.

The reason why we are interested in studying this class of systems is to capture discontinuity actions in the dynamics from either the controller or system nonlinearities. Although widely used, very few results are available to analyze most PLS. More precisely, one typically cannot guarantee stability, robustness, and performance properties of PLS designs. Rather, any such properties are inferred from extensive computer simulations.

In [4], we introduced an entirely new methodology to globally analyze symmetric unimodal limit cycles ${ }^{1}$ of relay feedback systems. The idea consisted in finding a quadratic Lyapunov function on a switching surface that can be used to prove that the associated Poincaré map is contracting in some sense.

This paper generalizes the ideas from [4] to globally analyze PLS. In a similar way, the main idea consists in finding quadratic Lyapunov functions on associated switching surfaces that can be used to prove that impact maps, i.e., maps from one switching surface to the next switching surface, are contracting in some sense. The notion of an impact map can be though as a generalization of a Poincare map. Impact maps are known to be "unfriendly" maps in the sense that they are highly nonlinear, multivalued, and not continuous. The novelty of this work comes from expressing impact maps induced by an LTI flow between two hyperplanes as linear transformations analytically parameterized by a scalar function of the state. Furthermore, level sets of this function are convex subsets of linear manifolds with dimension lower than that of the switching surfaces. This allows us to search for quadratic surface Lyapunov functions by solving sets of LMIs using efficient computational algorithms. Contractions of certain impact maps of the system can then be used to conclude about global stability, robustness, and performance of PLS.

In [1], we show that this new methodology can be used to not only globally analyze limit cycles but also equilibrium points of PLS. For that, we analyze on/off and saturation systems, including those with unstable nonlinearity sectors for which classical methods like Popov criterion, Zames-Falb criterion, IQCs, fail to analyze. Although this analysis methodology yields only sufficient criteria of stability, it has shown to be very successful in globally analyzing a large number of examples with a locally stable limit cycle or equilibrium point. In fact, it is still an open problem whether there exists an example with a globally stable limit cycle or equilibrium point that could not be successfully analyzed with this new methodology. Examples analyzed include systems of relative degree larger than one and of high dimension, for which no other analysis methodology could be applied.

${ }^{1} \mathrm{~A}$ limit cycle is unimodal if it only switches twice per cycle. 
We have shown [2, chapter 8] that this methodology can be efficiently applied to not only globally analyze stability of limit cycles and equilibrium points, but also robustness, and performance of PLS. This success in globally analyzing stability, robustness, and performance of certain classes of PLS has shown the power of this new methodology, and suggests its potential towards the analysis of larger and more complex PLS.

A more complete and detailed version of this paper has been submitted for publication [3].

\section{Motivation}

As discussed in introduction, there exist several tools to analyze PLS. One of the most important $[7,9,6]$ is based in constructing piecewise quadratic Lyapunov functions in the state space. There are, however, several drawbacks with this approach. First, piecewise quadratic Lyapunov functions in the state space cannot be constructed to analyze most limit cycles. Second, for most PLS, it is not possible to construct piecewise quadratic Lyapunov functions with just the given natural partition of the system. In order to improve flexibility a subdivision of partitions is typically necessary. The analysis method, however, is efficient only when the number of partitions required to prove stability is small. Example 4.1 in [2] shows that even for second order systems, the construction of piecewise quadratic Lyapunov functions can be computationally intractable due to the large number of partitions in the state space required for the analysis. Third, in general, for systems of order higher than 3 , it is extremely hard to obtain a refinement of partitions in the state-space to efficiently analyze PLS using piecewise quadratic Lyapunov functions. In fact, only a few and specific examples of PLS of order higher than 3 analyzed with this method have been reported. Finally, existence of piecewise quadratic Lyapunov functions implies exponential stability of the system. Thus, this approach cannot prove asymptotic stability of PLS when these are not exponentially stable.

The construction of piecewise quadratic Lyapunov functions for PLS proposed in $[7,9,6]$ imposes continuity of the the Lyapunov functions along switching surfaces. This means that the intersection of two Lyapunov functions with a switching surface-one from each side-defines a unique quadratic Lyapunov function on the switching surface. Therefore, we conclude that if there are piecewise quadratic Lyapunov functions for a certain PLS, then there are also quadratic Lyapunov functions on switching surfaces for that same PLS. Note that the converse is not true. For instance, piecewise quadratic Lyapunov functions cannot be constructed to analyze limit cycles. However, as demonstrated in [4], quadratic Lyapunov functions on switching surfaces exist and can be efficiently constructed to analyze limit cycles.

The purpose of this paper is to show how Lyapunov functions on switching surfaces can be efficiently constructed. We call these Quadratic Surface Lyapunov Functions. Since a PLS behaves linearly inside each cell, only one of the following three scenarios can happen to a trajectory entering a cell at some point $x_{0}$ on a switching surface (see figure 1 ):

1. The cell is unbounded and there exists a trajectory that will grow unbounded without ever switching. In this case, $x_{0}$ belongs to an unstable region of the PLS.

2. There is a locally stable equilibrium point in the cell and the trajectory will asymptotically converge to it without switching. If this is the case, $x_{0}$ belongs to a stable region of that equilibrium point.

3. The trajectory will switch in finite time.
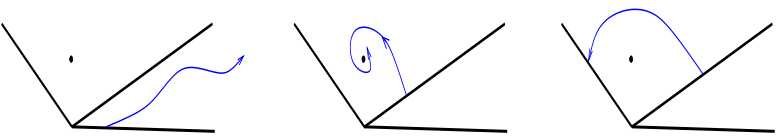

Figure 1: Possible scenarios for a trajectory entering a cell

There are several ways to check if scenario 1 can happen or not (see $[4,1])$. For now, assume that scenario 1 does not happen. If scenario 2 happens, we are done, i.e., the initial point $x_{0}$ is a stable point and so it does not require any further analysis. So, we are left with scenario 3 . This scenario raises several interesting questions: what happens to the trajectory after it switches? Will it switch again? And, will it converge to some equilibrium point or some limit cycle? These are the sort of questions we address in this paper. The idea is to start by analyzing individual maps from one switching surface to the next switching surface. Then, we show that the analysis of PLS can be reduced to the simultaneously analysis of different maps from one switching surface to another switching surface.

Analysis of nonlinear systems at manifolds has been used by many researchers for a while now. The so-called Poincaré map was introduced in order to reduce the study of an $n$ dimensional system to a discrete $n \Leftrightarrow 1$-dimensional system in a manifold (see, for example, [8] for an introduction to Poincaré maps). The problem with Poincaré maps is that they are typically nonlinear, not continuous, and multivalued. Thus, global analysis of PLS is rarely done using these maps. This paper explains how the difficulties inherent to Poincaré maps can be overcome and how they can be used to globally analyze PLS. We show that our results really work in the sense that a large number of examples of certain classes of PLS, that could not be analyzed by any other method, were successfully proven globally stable.

All the problems described above associated with the method proposed by $[9,7,6]$, based on piecewise quadratic Lyapunov functions, were not an issue in the classes of PLS analyzed so far using quadratic surface Lyapunov functions. First, quadratic surface Lyapunov functions can analyze both limit cycles [4] and equilibrium points [1]. Second, it was sufficient to consider only the natural partition of all PLS analyzed so far, with no extra complexity added. Note that none of examples in $[4,1]$ could be analyzed with just their natural partition using piecewise quadratic Lyapunov functions. Third, our new method scales well with the dimension of the system. And, finally, quadratic surface Lyapunov functions can be used to prove global asymptotic stability of PLS that are not exponentially stable (see example 4.3 in [1]). 


\section{Impact maps}

In order to analyze PLS using quadratic surface Lyapunov functions, we first need to understand the behavior of the system as this flows from one switching surface to the next switching surface. A useful notion that we will be using throughout this paper is that of impact map. An impact map is simply a map from one switching surface to the next switching surface. Only after we understand the nature of a single impact map can we look at a PLS as a whole, by combining all impact maps associated with the PLS, to conclude about stability, robustness, and performance properties of the system.

Consider the following affine linear time-invariant system

$$
\dot{x}=A x+B
$$

where $x \in \mathbb{R}^{n}, A \in \mathbb{R}^{n \times n}$, and $B \in \mathbb{R}^{n}$. Note that we are not imposing any kind of restrictions on $A$. Matrix $A$ is allowed to have stable, unstable, and pure imaginary eigenvalues. Assume (1) is part of some PLS, and that (1) is defined on some open polytopical set $X \subset \mathbb{R}^{n}$. Assume also a trajectory just arrived to a subset of the boundary ${ }^{2}$ of $X$ belonging to

$$
S_{0}=\left\{x \in \mathbb{R}^{n}: C_{0} x=d_{0}\right\}
$$

and the system switches to (1). In this paper, we are interested in studying the impact map from some subset of $S_{0}$ to some subset of

$$
S_{1}=\left\{x \in \mathbb{R}^{n}: C_{1} x=d_{1}\right\}
$$

also in the boundary of $X$. In this scenario, some subsets of $S_{0}$ and $S_{1}$ are switching surfaces of the PLS.

By a solution of (1) we mean a function $x$ defined on $[0, t]$, with $x(0) \in S_{0}, x(t) \in S_{1}, x(\tau) \in \bar{X}$ on $[0, t]^{3}$, and satisfying (1). In this case, $t$ is a switching time of the solution $x$ of (1) and we say a switch occurs at $x(t)$.

Let $S_{0}^{d}$ be some polytopical subset of $S_{0}$ where any trajectory starting at $S_{0}^{d}$ satisfies $x(t) \in S_{1}$, for some finite $t \geq 0$, and $x(\tau) \in \bar{X}$ on $[0, t]$. Let also $S_{1}^{a} \subset S_{1}$ be the set of those points $x_{1}=x(t)$. The set $S_{1}^{a}$ can be seen as the image set of $S_{0}^{d}$. We call $S_{0}^{d}$ the departure set in $S_{0}$ and $S_{1}^{a}$ the arrival set in $S_{1}$.

We are interested in studying the impact map, induced by (1), from $x_{0} \in S_{0}^{d}$ to $x_{1} \in S_{1}^{a}$. Since both $x_{0}$ and $x_{1}$ belong to switching surfaces, they can be parameterized in their respective hyperplanes. For that, let

$$
\begin{aligned}
& x_{0}=x_{0}^{*}+\Delta_{0} \\
& x_{1}=x_{1}^{*}+\Delta_{1}
\end{aligned}
$$

where $x_{0}^{*} \in S_{0}, x_{1}^{*} \in S_{1}$, and $\Delta_{0}, \Delta_{1}$ are any vectors such that $\Delta_{0} \in S_{0}^{d} \Leftrightarrow x_{0}^{*}$ and $\Delta_{1} \in S_{1}^{a} \Leftrightarrow x_{1}^{*}$. In this case, $C_{0} \Delta_{0}=$ $C_{1} \Delta_{1}=0$. Define also $x_{0}^{*}(t)$ as the trajectory of (1), starting at $x_{0}^{*}$, for all $t \geq 0$. The impact map of interest reduces to the map from $\Delta_{0}$ to $\Delta_{1}$ (see figure 2).

Note that, in general, the impact map from $\Delta_{0} \in S_{0}^{d} \Leftrightarrow x_{0}^{*}$ to $\Delta_{1} \in S_{1}^{a} \Leftrightarrow x_{1}^{*}$ defined above is not continuous and it is multivalued. This is illustrated in example 3.1 in [1]

\footnotetext{
${ }^{2}$ The boundary of $X$ is the set of all limit points $p$ of $X$ such that $p \notin X$.

${ }^{3} \bar{X}$ denotes the closure of $X$, i.e, the set $\bar{X}=X \cup$ $\{p \mid p$ is a limit point of $X\}$.
}

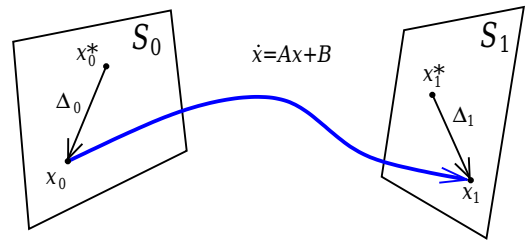

Figure 2: Impact map from $\Delta_{0} \in S_{0}^{d} \Leftrightarrow x_{0}^{*}$ to $\Delta_{1} \in S_{1}^{a} \Leftrightarrow x_{1}^{*}$

Definition 3.1 Let $x(0)=x_{0}^{*}+\Delta_{0}$. Define $t_{\Delta_{0}}$ as the set of all times $t_{i} \geq 0$ such that the trajectory $x(t)$ with initial condition $x(0)$ satisfies $C_{1} x\left(t_{i}\right)=d_{1}$ and $x(t) \in \bar{X}$ on $\left[0, t_{i}\right]$. Define also the set of expected switching times of the impact map from $\Delta_{0} \in S_{0}^{d} \Leftrightarrow x_{0}^{*}$ to $\Delta_{1} \in S_{1}^{a} \Leftrightarrow x_{1}^{*}$ as

$$
\mathcal{T}=\left\{t \mid t \in t_{\Delta_{0}}, \Delta_{0} \in S_{0}^{d} \Leftrightarrow x_{0}^{*}\right\}
$$

For an impact map, once an initial condition $\Delta_{0} \in S_{0}^{d} \Leftrightarrow x_{0}^{*}$ is given, in order to find an image $\Delta_{1} \in S_{1}^{a} \Leftrightarrow x_{1}^{*}$, we must first find an associated switching time $t$. Solving for $t$, however, involves solving a transcendental equation. Solution to such equations cannot, in general, be written in closed form, and numerical procedures are typically the only way to solve for $t$. Once a switching time is found, we can finally find the corresponding $\Delta_{1}$.

Thus, in general, impact maps are highly nonlinear, multivalued, and not continuous. This "non-friendly" nature of impact maps is the main reason why global analysis of PLS has not been done before using quadratic surface Lyapunov functions. The following result, however, shows that this map is not as "bad" as it looks, and opens the door to analysis of PLS in switching surfaces.

Theorem 3.1 Assume $C_{1} x_{0}^{*}(t) \neq d_{1}$ for all $t \in \mathcal{T}$. Define

and let

$$
w(t)=\frac{C_{1} e^{A t}}{d_{1} \Leftrightarrow C_{1} x_{0}^{*}(t)}
$$

$$
H(t)=e^{A t}+\left(x_{0}^{*}(t) \Leftrightarrow x_{1}^{*}\right) w(t)
$$

Then, for any $\Delta_{0} \in S_{0}^{d} \Leftrightarrow x_{0}^{*}$ there exists a $t \in \mathcal{T}$ such that the impact map is given by

$$
\Delta_{1}=H(t) \Delta_{0}
$$

Such $t \in t_{\Delta_{0}}$ is the switching time associated with $\Delta_{1}$.

This theorem says that maps between switching surfaces, induced by an LTI flow, can be represented as linear transformations analytically parameterized by a scalar function of the state. At first, equation (2) may not seem of great help in analyzing the impact map from $\Delta_{0}$ to $\Delta_{1}$. There, $\Delta_{1}$ is a linear function of $\Delta_{0}$ and a nonlinear function of $t$, the switching time associated with $\Delta_{0}$ and $\Delta_{1}$. The switching time, however, is a function of $\Delta_{0}$. A transcendental equation needs to be solved in order to find $t$. Thus, by this reasoning, it seems (2) is saying that $\Delta_{1}$ is a nonlinear function of $\Delta_{0}$. But, that we already knew.

This is, however, just one way of thinking about (2). Fortunately, there are other ways to approach equation (2). Assume, for now, the switching time $t$ is fixed. The result: the impact map (2) would be linear! But, what does it mean having the 
switching time $t$ fixed? In other words, what are the set of points $x_{0}^{*}+\Delta_{0}$ in the switching surface $S_{0}$ such that every point in that set has a switching time of $t$ ? In that set, the impact map (2) is linear.

It turns out that the set of points in $S_{0}^{d}$ that have a switching time of $t$ is a convex subset of a linear manifold of dimension $n \Leftrightarrow 2$ (see figure 3). Let $S_{t}$ be that set, that is, the set of points $x_{0}^{*}+\Delta_{0} \in S_{0}^{d}$ such that $t \in t_{\Delta_{0}}$. In other words, a trajectory starting at $x_{0} \in S_{t}$ satisfies both $x(\tau) \in \bar{X}$ on $[0, t]$, and $C_{1} x(t)=d_{1}$. Note that since the impact map is multivalued, a point in $S_{0}^{d}$ may belong to more than one set $S_{t}$.

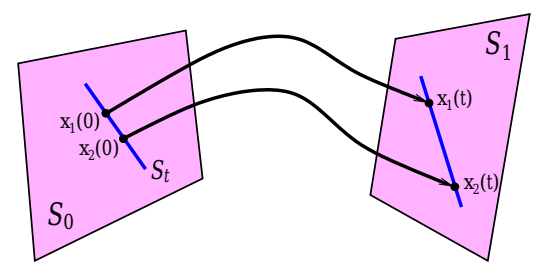

Figure 3: Every point in $S_{t}$ has a switching time of $t$

Note also that, as $t \in \mathcal{T}$ changes, $S_{t}$ covers every single point of $S_{0}^{d}$, i.e., $S_{0}^{d}=\left\{x \mid x \in S_{t}, t \in \mathcal{T}\right\}$. This follows since every point $\Delta_{0} \in S_{0}^{d} \Leftrightarrow x_{0}^{*}$ can switch for the first time at $S_{1}^{a}$, and therefore $t_{\Delta_{0}}$ is always a nonzero set. These results can all be summarized in the following corollary.

Corollary 3.1 Under the assumptions of theorem 3.1, for a given $t \in \mathcal{T}$, the impact map from $\Delta_{0} \in S_{t} \Leftrightarrow x_{0}^{*}$ to $\Delta_{1} \in$ $S_{1} \Leftrightarrow x_{1}^{*}$, given by $\Delta_{1}=H(t) \Delta_{0}$, is a linear map. Moreover, $S_{t}$ is a subset of a linear manifold of dimension $n \Leftrightarrow 2$, and $S_{0}^{d}=\left\{x \mid x \in S_{t}, t \in \mathcal{T}\right\}$.

As we will see in section 4, this result is fundamental in the analysis of PLS using quadratic surface Lyapunov functions. It allows us to find conditions in the form of LMIs that, when satisfied, guarantee stability, robustness, and performance of PLS.

Before moving onto the proofs of the above results, it is important to understand the meaning of the assumption in theorem 3.1. This says the trajectory $x_{0}^{*}(t)$ cannot intersect the switching surface $S_{1}$ for all $t \in \mathcal{T}$. With a careful choice of $x_{0}^{*} \in S_{0}$ (the initial condition of $x_{0}^{*}(t)$ ), there are many cases when this assumption is always satisfied, as explained in [1].

There are, however, cases where no choice of $x_{0}^{*} \in S_{0}$ satisfies the assumption. Or, in other cases, $x_{0}^{*}$ is fixed a priori (like in RFS [4], where the location of $x^{*}$ in $S_{0}$ cannot be freely chosen), and it may not satisfy the assumption. In these worst case scenarios, there is at least one $t_{s} \in \mathcal{T}$ such that $C_{1} x_{0}^{*}\left(t_{s}\right)=d_{1}$. This does not mean we cannot obtain the desired linear representation for the impact map. For some PLS, like RFS [4], $w(t)$ is defined via continuation, at some $t=t_{s}$, and the theorem follows. If this is not the case, the theorem needs to be slightly modified. Basically, at $t=t_{s}$, the impact map can still be written as a linear transformation but parameterized by another variable at $t_{s}$, i.e., $\Delta_{1}=H_{s}\left(t_{s}, \delta\right) \Delta_{0}$, with $\Delta_{0} \in S_{t_{s}}$.

Proof of theorem 3.1: We start by expressing $\Delta_{1}$ as function Proceedings of the European Control Conference 2001 of $\Delta_{0}$ and $t$, the switching time associated with $\Delta_{1}$. Let $x(0)=$ $x_{0} \in S_{0}^{d}$. Integrating the differential equation (1) gives

$$
x_{1}=e^{A t} x_{0}+\int_{0}^{t} e^{A(t-\tau)} B d \tau
$$

Since $x_{i}=x_{i}^{*}+\Delta_{i}, i=0,1$,

$$
\begin{aligned}
\Delta_{1} & =e^{A t} \Delta_{0}+e^{A t} x_{0}^{*}+\int_{0}^{t} e^{A(t-\tau)} B d \tau \Leftrightarrow x_{1}^{*} \\
& =e^{A t} \Delta_{0}+x_{0}^{*}(t) \Leftrightarrow x_{1}^{*}
\end{aligned}
$$

From the fact $C_{1} \Delta_{1}=0$ and $C_{1} x_{1}^{*}=d_{1}$ we get

$$
C_{1} e^{A t} \Delta_{0}=d_{1} \Leftrightarrow C_{1} x_{0}^{*}(t)
$$

Since, by assumption, $C_{1} x_{0}^{*}(t) \neq d_{1}$ for all $t \in \mathcal{T}$, the last expression can be written as

$$
w(t) \Delta_{0}=1
$$

which means $\Delta_{1}$ reduces to $e^{A t} \Delta_{0}+\left(x_{0}^{*}(t) \Leftrightarrow x_{1}^{*}\right) w(t) \Delta_{0}$

Note that if $A$ is invertible, $x_{0}^{*}(t)$ can be written as $x_{0}^{*}(t)=$ $e^{A t}\left(x_{0}^{*}+A^{-1} B\right) \Leftrightarrow A^{-1} B$.

Proof of corollary 3.1: The only thing left to prove is that $S_{t}$ is a subset of a linear manifold of dimension $n \Leftrightarrow 2$. Let $x_{0}=x_{0}^{*}+$ $\Delta_{0} \in S_{t}$. Since $C_{1} x(t)=d_{1}, \Delta_{0}$ must satisfy equation (3), and $C_{0} \Delta_{0}=0$ since $\Delta_{0} \in S_{0} \Leftrightarrow x_{0}^{*}$, which are both linear equalities. $\Delta_{0}$ also satisfies a set of linear inequalities from the fact that $x_{0} \in S_{0}^{d}, x(t) \in S_{1}^{a}$, and $x(\tau) \in \bar{X}$ on $[0, t]$. Therefore, $S_{t} \Leftrightarrow x_{0}^{*}$ has at most dimension $n \Leftrightarrow 2$ and is linear.

\section{Quadratic surface Lyapunov functions}

Construction of Lyapunov functions for nonlinear systems is, and has been, a difficult, and sometimes, frustrating task. As explained before, there has been some results in constructing piecewise quadratic Lyapunov functions for PLS. Although these results are able to analyze equilibrium points of certain classes of PLS, many important PLS cannot be analyzed this way because either they have limit cycles or the method is computationally too expensive.

An alternative to constructing Lyapunov functions in the state space is to construct Lyapunov functions on switching surfaces. Define then two quadratic Lyapunov functions on the switching surfaces $S_{0}^{d}$ and $S_{1}^{a}$. Respectively, let $V_{0}$ and $V_{1}$ be given by

$$
V_{i}(x)=x^{\prime} P_{i} x \Leftrightarrow 2 x^{\prime} g_{i}+\alpha_{i}
$$

where $P_{i}>0$, for $i=0,1$. These are Lyapunov candidates defined of the switching surfaces with parameters $P_{i}>0, g_{i}$, and $\alpha_{i}$, to be found.

Next, we want to show an impact map from $S_{0}^{d} \subset S_{0}$ to $S_{1}^{a} \subset$ $S_{1}$ is contracting in some sense. In particular, an impact map is quadratically stable if there exist $P_{i}>0, g_{i}, \alpha_{i}$ such that

$$
V_{1}\left(\Delta_{1}\right)<V_{0}\left(\Delta_{0}\right) \text { for all } \Delta_{0} \in S_{0}^{d} \Leftrightarrow x_{0}^{*}
$$

Let $P>0$ on $S$ stand for $x^{\prime} P x>0$ for all nonzero $x \in S$. As a short hand, we will be using $H_{t}$ for $H(t)$ and $w_{t}$ for $w(t)$. The following theorem takes advantage of the results from section 3 to derive a set of matrix inequalities equivalent to condition (6). 1360 
Theorem 4.1 Define

$$
R(t)=P_{0} \Leftrightarrow H_{t}^{\prime} P_{1} H_{t} \Leftrightarrow 2\left(g_{0} \Leftrightarrow H_{t}^{\prime} g_{1}\right) w_{t}+w_{t}^{\prime} \alpha w_{t}
$$

where $\alpha=\alpha_{0} \Leftrightarrow \alpha_{1}$. The impact map from $\Delta_{0} \in S_{0}^{d} \Leftrightarrow x_{0}^{*}$ to $\Delta_{1} \in S_{1}^{a} \Leftrightarrow x_{1}^{*}$ is a contraction if there exist $P_{0}, P_{1}>0$ and $g_{0}, g_{1}, \alpha$ such that

$$
R(t)>0 \text { on } S_{t} \Leftrightarrow x_{0}^{*}
$$

for all expected switching times $t \in \mathcal{T}$.

Basically, all this theorem does is substitute (2) in (6), and use both facts that the map $\Delta_{0}$ to $\Delta_{1}$ is linear in $S_{t}$ and that, as $t$ ranges over $\mathcal{T}, S_{t}$ covers every point in $S_{0}^{d}$.

\subsection{Approximation by a set of LMIs}

There are many ways to approximate condition (7) with a set of LMIs, which can be efficiently solved using available software. By definition, condition (7) is equivalent to $\Delta_{0}^{\prime} R(t) \Delta_{0}>0$ for all $\Delta_{0} \in S_{t} \Leftrightarrow x_{0}^{*}$. A more conservative condition results when $\Delta_{0}$ is relaxed:

$$
\Delta_{0}^{\prime} R(t) \Delta_{0}>0 \text { for all } \Delta_{0} \in S_{0}^{d} \Leftrightarrow x_{0}^{*}
$$

If this condition is satisfied then (7) follows since $S_{t} \subset S_{0}^{d}$. A trivial way to obtain a set of LMIs is to further relax the constraints on $\Delta_{0}$. On one hand, this results in a more conservative condition. On the other hand, such condition is computationally efficient.

Corollary 4.1 The impact map from $\Delta_{0} \in S_{0}^{d} \Leftrightarrow x_{0}^{*}$ to $\Delta_{1} \in$ $S_{1}^{a} \Leftrightarrow x_{1}^{*}$ is a contraction if there exist $P_{0}, P_{1}>0$ and $g_{0}, g_{1}, \alpha$ such that

$$
R(t)>0 \text { on } S_{0} \Leftrightarrow x_{0}^{*}
$$

for all expected switching times $t \in \mathcal{T}$.

This result uses the ideas from the previous section to show that the problem of quadratic stability of an impact map reduces to the solution of a infinite dimensional set of LMIs. As shown in several examples in [4] and [1], although condition (8) is more conservative than (7), in many situations this is enough to efficiently and successfully globally analyze PLS. Condition (8) can be written as an equivalent set of LMIs by noticing that impact maps are $n \Leftrightarrow 1$-dimensional maps. See [1] for details.

It is possible to make condition (8) less conservative at a cost of increase computations. This condition takes only into account that $\Delta_{0} \in S_{0} \Leftrightarrow x_{0}^{*}$. In [1], we explain how to approximate condition (7) with less conservative sets of LMIs than (8).

\subsection{Proof of Results}

Proof of theorem 4.1: From (6) and using (2) and corollary 3.1, we have

$$
\begin{aligned}
& \Delta_{1}^{\prime} P_{1} \Delta_{1} \Leftrightarrow 2 \Delta_{1}^{\prime} g_{1}+\alpha_{1}<\Delta_{0}^{\prime} P_{0} \Delta_{0} \Leftrightarrow 2 \Delta_{0}^{\prime} g_{0}+\alpha_{0} \\
\Leftrightarrow & \Delta_{0}^{\prime}\left(P_{0} \Leftrightarrow H_{t}^{\prime} P_{1} H_{t}\right) \Delta_{0} \Leftrightarrow 2 \Delta_{0}^{\prime}\left(g_{0} \Leftrightarrow H_{t}^{\prime} g_{1}\right)+\alpha>0
\end{aligned}
$$

for all $\Delta_{0} \in S_{t} \Leftrightarrow x_{0}^{*}$. Finally, using (4) the result follows.

Proof of corollary 4.1: The result follows since $S_{t} \subset S_{0}$.

\section{Global analysis of PLS}

We have seen how global analysis of a single impact map can be done using quadratic Lyapunov functions defined on switching surfaces. The next question is how to combine different impact maps associated with a PLS to globally analyze the system? There are several different issues that arise when analyzing PLS using quadratic surface Lyapunov functions. This section explains the three main steps to globally analyze a PLS. These consist on (1) characterization of impact maps, (2) definition of quadratic Lyapunov functions at switching surfaces, and finally (3) solution of stability conditions. These steps consist on (see [1] for more details):

\section{Step 1: Impact Maps}

1. Identification of all impact maps associated with the PLS. If the system has $m$ switching surfaces then there are at the most $2 m^{2}$ impact maps. The actual number of impact maps required to analyze the system is typically smaller due to certain properties of a system, like symmetry (see relay feedback systems [4] or saturation systems [1]) or just the fact that not all switches are possible.

2. Characterization of domains of impact maps.

3. Necessary conditions. Certain conditions need to be checked in order to guarantee that a trajectory, starting in a switching surface, does not grow unbounded without switching (as in the left of figure 1).

4. Linear decomposition. For each impact map, we need to find an $x_{0}^{*}$ belonging to the switching surface where the domain of an impact is defined, such that the assumption of theorem 3.1 is satisfied. If this is not possible, the switching times where the assumption is not satisfied need to be characterized, and then proceed as explained in [3].

\section{Step 2: Quadratic Surface Lyapunov Functions}

1. Define all quadratic surface Lyapunov functions on the respective domains of impact maps. There are at the most $2 m$ Lyapunov functions, where $m$ is the number of switching surfaces of the PLS.

2. Constraints on quadratic surface Lyapunov functions: continuity issues, and limit cycles and equilibrium points

3. Switching times and switching time bounds. For each impact map, we have a quadratic inequality that must be satisfied for all expected switching times $\mathcal{T}$ associated with the impact map. In many situations, however, it is not necessary to check if a quadratic inequality is satisfied for all $t \in \mathcal{T}$, but it is enough that this is true only on a bounded subset of $\mathcal{T}$.

\section{Step 3: Stability Conditions}

1. Write stability conditions as LMIs using corollary 4.1, for each impact map.

2. Improvements of stability conditions. If there is no feasible solution to the LMIs described in the previous item, we can use less conservative conditions (see [1]).

3. An alternative to solving all the LMIs described above is to add LMIs until all quadratic constraints are satisfied, since checking if a quadratic inequality is satisfied is much easier than solving the correspondent LMIs.

Before attempting to analyze general classes of PLS with quadratic surface Lyapunov functions using this algorithm, it is important to fully understand in detail each of the steps in the algorithm. For that purpose, we have analyzed several classes 
of PLS by increasing order of complexity [4, 1]. Each of these classes was carefully chosen to (1) separately deal with different issues in each step of the algorithm and (2) to illustrate with examples the efficiency of this new methodology. By increasing complexity, we first analyzed relay feedback systems [4], then on/off systems [1], and finally saturation systems [1]. The success in globally analyzing a large number of examples of these classes of PLS demonstrated the potential of these new ideas in globally analyzing other, more complex classes of PLS.

The reasons for analyzing these particular classes of PLS are the following. In relay feedback systems [4], we analyzed limit cycles. The choice to first analyze this class of PLS was based on the fact that, for symmetric unimodal limit cycles, there is only a single impact map that needs to be studied. This means that global analysis of symmetric unimodal limit cycles of relay feedback systems is simply a special case of theorem 4.1.

In the analysis of on/off systems [1], we explained (1) how this new methodology is used to globally analyze equilibrium points and (2) how more than one impact map is simultaneously analyzed. Finally, with saturation systems [1] we showed how to deal with multiple switching surfaces.

Analysis of other, more complex classes of PLS can be done using a combination of the ideas discussed above. A PLS can have limit cycles or equilibrium points, and several switching surfaces that may or may not contain equilibrium points or intersect limit cycles. After a characterization of all relevant impact maps, these can be simultaneously analyzed to conclude about the stability the system. The main feature remains the fact that quadratic stability of an impact map can be easily checked by solving a set of LMIs, as explained in sections 3 and 4 . The latest version of the above algorithm can be obtained at [5].

\section{Conclusions}

Motivated by the need of better, more general, and more efficient global analysis tools for certain classes of hybrid systems, this paper developed an entirely new constructive analysis methodology for PLS using impact maps and quadratic surface Lyapunov functions. This methodology consists in inferring global properties of PLS solely by studying their behavior at switching surfaces associated with PLS. The main idea is to construct quadratic surface Lyapunov functions to show that maps between switching surfaces are contracting in some sense. These results are based on the discovery that maps induced by an LTI flow between two switching surfaces can be represented as linear transformations analytically parameterized by a scalar function of the state. Furthermore, level sets of this function are convex subsets of linear manifolds. This representation allows the search for quadratic Lyapunov functions on switching surfaces to be done by simply and efficiently solving a set of LMIs.

The success and power of this new methodology has been demonstrated in globally analyzing equilibrium points and limit cycles of several classes of piecewise linear systems (PLS): relay feedback systems, on/off systems, and saturation systems. A large number of examples of these classes of PLS with a locally stable limit cycle or equilibrium point were successfully globally analyzed using this analysis methodology. In fact, it is still an open problem whether there exists an example with a globally stable limit cycle or equilibrium point that could not be successfully analyzed with this new methodology.

\section{Acknowledgments}

This research was supported in part by the Portuguese Science and Technology Foundation under the program "PRAXIS XXI", by the NSF under grants ECS-9410531, ECS-9796099, ECS-9796033, and ECS-9612558, and by the AFOSR under grants F49620-96-1-0123, F49620-00-1-0096, F49620-950219, and F49620-99-1-0320.

\section{References}

[1] Jorge M. Gonçalves. Global analysis of piecewise linear systems using impact maps and quadratic surface Lyapunov functions: application to on/off and saturation systems. Submited for publication, IEEE Transactions on Automatic Control.

[2] Jorge M. Gonçalves. Constructive Global Analysis of Hybrid Systems. PhD thesis, Massachusetts Institute of Technology, Cambridge, MA, September 2000.

[3] Jorge M. Gonçalves, Alexandre Megretski, and Munther A. Dahleh. Global analysis of piecewise linear systems using impact maps and quadratic surface Lyapunov functions: general theory. Submited for publication, IEEE Transactions on Automatic Control.

[4] Jorge M. Gonçalves, Alexandre Megretski, and Munther A. Dahleh. Global stability of relay feedback systems. IEEE Transactions on Automatic Control, 46(4), April 2001.

[5] Jorge M. Gonçalves' web page. http: / / web.mit.edu/jmg/www/.

[6] Arash Hassibi and Stephen Boyd. Quadratic stabilization and control of piecewise linear systems. In ACC, Philadelphia, Pennsylvania, June 1998.

[7] Mikael Johansson and Anders Rantzer. Computation of piecewise quadratic Lyapunov functions for hybrid systems. IEEE Transactions on Automatic Control, 43(4):555-559, April 1998.

[8] Hassan K. Khalil. Nonlinear Systems. Prentice Hall, N.J., 2nd edition, 1996.

[9] Stefan Pettersson and Bengt Lennartson. An LMI approach for stability analysis of nonlinear systems. In EEC, Brussels, Belgium, July 1997. 